\title{
The globular cluster systems of Abell 1185^
}

\author{
M. J. West ${ }^{1}$, A. Jordán ${ }^{2}$, J. P. Blakeslee ${ }^{3}$, P. Côté ${ }^{3}$, M. D. Gregg ${ }^{4,5}$, M. Takamiya ${ }^{6}$, and R. O. Marzke ${ }^{7}$ \\ ${ }^{1}$ European Southern Observatory, Alonso de Cordova 3107, Vitacura, Santiago, Chile \\ e-mail: mwest@eso.org \\ 2 Departamento de Astronomía y Astrofísica, Pontificia Universidad Católica de Chile, Casilla 306, Santiago 22, Chile \\ e-mail: ajordan@astro.puc.cl \\ ${ }^{3}$ Herzberg Institute of Astrophysics, National Research Council of Canada, Victoria, BC V9E 2E7, Canada \\ e-mail: [john.blakeslee; patrick.cote]@nrc-cnrc.gc.ca \\ ${ }^{4}$ Institute for Geophysics and Planetary Physics, Lawrence Livermore National Laboratory, L-413, Livermore, CA 94550, USA \\ e-mail: gregg@igpp.ucllnl.org \\ 5 Department of Physics, University of California, Davis, CA 956160, USA \\ e-mail: gregg@igpp.ucllnl.org \\ 6 Department of Physics and Astronomy, University of Hawaii, Hilo, HI 96720, USA \\ e-mail: mtakamiya@hawaii.edu \\ 7 Department of Physics and Astronomy, San Francisco State University, San Francisco, CA 94132-4163, USA \\ e-mail: marzke@stars.sfsu.edu
}

Received 15 October 2010 / Accepted 26 January 2011

\section{ABSTRACT}

\begin{abstract}
We examine the properties of a previously discovered population of globular clusters in the heart of the rich galaxy cluster Abell 1185 that might be intergalactic in nature. Deep images obtained with the Advanced Camera for Surveys (ACS) aboard Hubble Space Telescope (HST) confirm the presence of $\sim 1300$ globular clusters brighter than $I_{F 814 W} \simeq 27.3$ mag in a field devoid of any large galaxies. The luminosities and colors of these objects are found to be similar to those of metal-poor globular clusters observed in many galaxies to date. Although a significant fraction of the detected globular clusters undoubtedly reside in the outer halos of galaxies adjacent to this field, detailed modeling of their distribution suggests that the majority of these objects are likely to be intergalactic, in the sense that they are not gravitationally bound to any individual galaxy. We conclude that the true nature and origin of the globular cluster population in the core of A1185 - galactic residents or intergalactic wanderers - remains uncertain, and suggest how future observation could resolve this ambiguity.
\end{abstract}

Key words. galaxies: elliptical and lenticular, cD - galaxies: formation - galaxies: interactions - globular clusters: general galaxies: clusters: individual: Abell 1185

\section{Introduction}

Globular clusters are ubiquitous in galaxies, found in all but the smallest dwarfs (see, e.g., Fig. 3 of Peng et al. 2008). For this reason they have long played a key role in astronomers' quest to understand the origin and evolution of galaxies (see West et al. 2004; and Brodie \& Strader 2006, for reviews).

However, a growing body of observational evidence also hints at the existence of a population of intergalactic globular clusters (hereafter IGCs) that reside outside of galaxies. Half a century ago, van den Bergh (1958) speculated that "about one third of all the globular clusters are of the intergalactic type" and numerous studies since then have lent support to the idea that at least some globular clusters are not gravitationally bound to individual galaxies (e.g., Muzzio et al. 1984; White 1987; West et al. 1995; Bassino et al. 2003; Hilker 2001; Jordán et al. 2003; Williams et al. 2007; Schuberth et al. 2008; Coenda et al. 2009; Gregg et al. 2009; Lee et al. 2010; Peng et al. 2011).

* Based on observations with the NASA/ESA Hubble Space Telescope obtained at the Space Telescope Science Institute (STScI), which is operated by the Association of Universities for Research in Astronomy, Inc., under NASA contract NAS 5-26555.
Little is known at present about the nature or origin of IGCs. One possibility is that they are normal globular clusters that were stripped from galaxies during gravitational interactions, the detritus of cosmic evolution. Globular clusters, being among the densest stellar systems, are likely to survive the partial or even complete disruption of their parent galaxy, resulting in an ever-growing population of orphaned star clusters that roam the space between galaxies. Alternatively, some globular clusters might have been born in intergalactic space without ever residing in galaxies, formed perhaps from the gravitational instability of primordial density fluctuations (e.g., Peebles \& Dicke 1968; Peebles 1984; West 1993; Yahagi \& Bekki 2005; Griffen et al. 2010, or thermal instabilities in cooling flows and other gas-rich environments (e.g., Fabian et al. 1984; Fall \& Rees 1985; Cen 2001; Griffen et al. 2010).

Whatever their origin, IGCs are likely to be most abundant in the extreme environments of galaxy clusters, where tidal forces and collisions between galaxies can pluck globular clusters from their halos or the dense local conditions might have allowed the birth of massive star clusters from intergalactic gas. Unfortunately, searches for IGCs in galaxy clusters are hindered by the fact that most have a giant elliptical at their center where the density of IGCs is expected to be greatest, making it difficult 


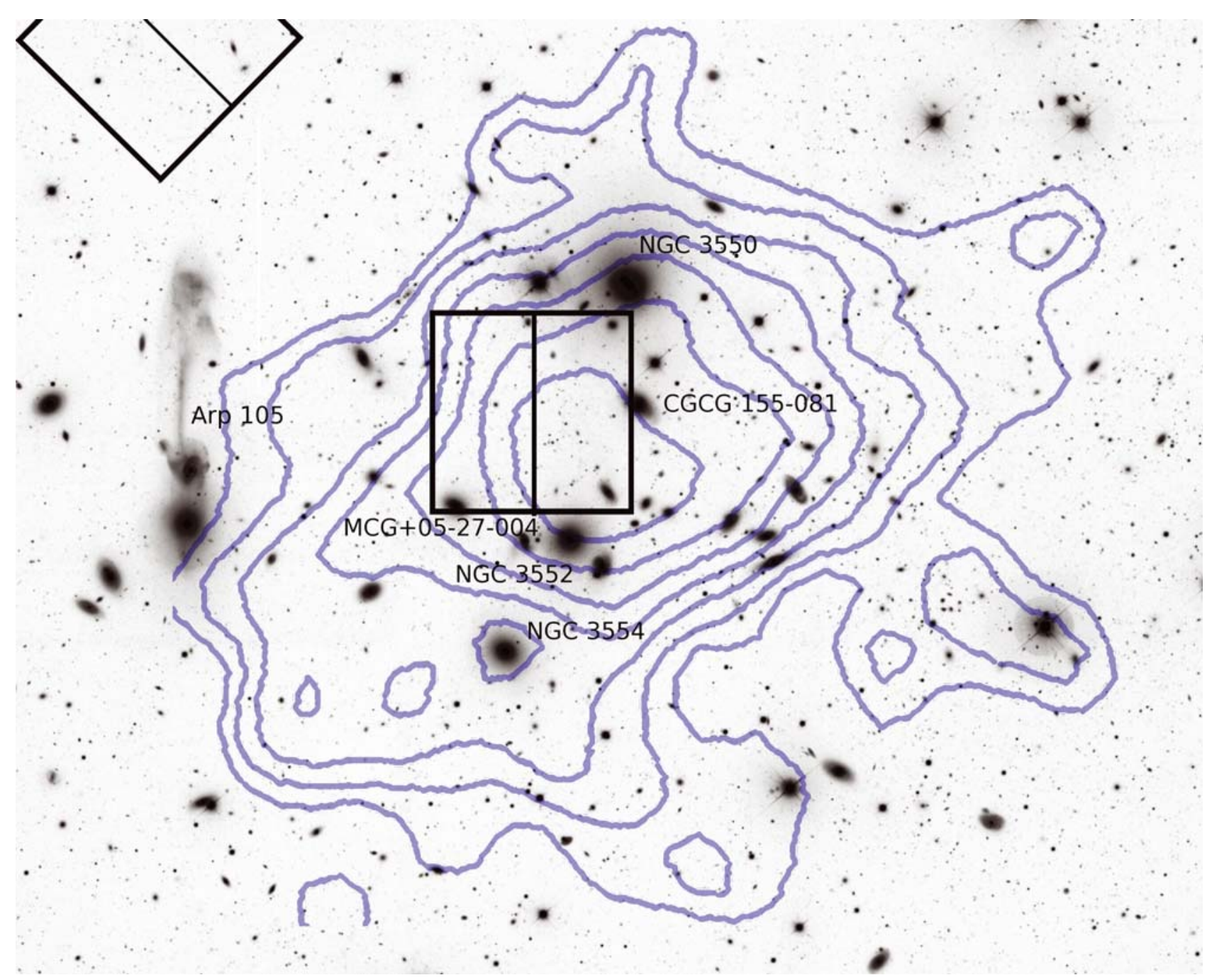

Fig. 1. A composite $B V R$ image of the rich cluster A1185 obtained with the CFH12k camera on CHFT (see Andreon et al. 2007) showing the locations of our ACS fields. The field of view is $\sim 20^{\prime} \times 16^{\prime}$, corresponding to $\sim 0.8 \mathrm{Mpc} \times 0.64 \mathrm{Mpc}$ at the distance of A1185. North is up and East is to the left. Our primary ACS field is located just below the brightest cluster galaxy, NGC 3550. A second ACS field located $\sim 9^{\prime}(\sim 350 \mathrm{kpc})$ to the northeast was used as a control field, as discussed in the text. Several of the brightest galaxies near the ACS field are identified, including the spectacular interacting galaxy pair Arp 105. X-ray contours from Mahdavi et al. (1996) based on Einstein observations are overlaid on the image.

to distinguish genuine IGCs from the galaxy's intrinsic globular cluster population.

An exception is Abell $1185(z=0.033)$, a rarity among low-redshift galaxy clusters because its brightest member galaxy (NGC 3550) is offset by $\sim 150 \mathrm{kpc}$ from the peak of the X-ray gas emission that presumably marks the centre of the cluster mass distribution (Mahdavi et al. 1996). Hence, A1185 offers an ideal opportunity to search directly for IGCs in the core of a relatively nearby cluster of galaxies. Tentative evidence of such a population was presented by Jordán et al. (2003), who detected 99 candidate IGCs in HST/WFPC2 images of a field in the center of A1185. However, their data suffered from incompleteness at magnitudes fainter than $I_{F 814 W} \simeq 25.5 \mathrm{mag}$, allowing only the brightest $\sim 10 \%$ of the expected globular cluster population to be detected at the distance of A1185.

In this paper, we analyze deep $F 555 W$ and $F 814 W$ ACS images of the core of A1185. We confirm the presence of a substantial population of globular clusters in this field and extend the results of Jordán et al. (2003) by presenting the first observations of the luminosity function and color distribution of these objects. The observations and data reductions are described in
Sect. 2 . Results from our analysis are presented in Sect. 3, and their implications are discussed in Sect. 4. We assume a standard cosmology with $H_{0}=72 \mathrm{~km} \mathrm{~s}^{-1} \mathrm{Mpc}^{-1}$ and a distance modulus $m-M \simeq 35.7 \mathrm{mag}$ to A1185. At this distance, an angle of $1^{\prime}$ subtends a projected linear scale of $65 \mathrm{kpc}$, and the $0.05^{\prime \prime}$ pixel scale of the ACS/WFC corresponds to a physical scale of $32 \mathrm{pc}$.

\section{Observations and data reductions}

Deep images of a field near the location of the peak X-ray emission in A1185 $\left(\mathrm{RA}=11^{\mathrm{h}} 10^{\mathrm{m}} 46^{\mathrm{s}}\right.$, Dec $\left.=+28^{\circ} 43^{\prime} 57^{\prime \prime}\right)$ were obtained using the ACS wide field channel (WFC) onboard HST as part of program GO-9438, which was allocated 15 orbits of time (see Figs. 1 and 2). Two filters were used, $F 555 W$ and $F 814 W$ (roughly equivalent to Johnson $V$ and Cousins $I$ ), with total exposure times of $15540 \mathrm{~s}$ and $23160 \mathrm{~s}$, respectively. The longer integration time of the $I_{F 814 W}$ images was chosen to ensure complete detection of the brightest half of the globular cluster luminosity function (GCLF) at the distance of A1185, which is assumed to have a Gaussian distribution with a peak at $M_{V} \simeq-7.4 \mathrm{mag}$ (e.g., Harris 2001; Jordán et al. 2007) and hence should occur at an apparent magnitude $I_{F 814 \mathrm{~W}} \simeq 27.3 \mathrm{mag}$ 


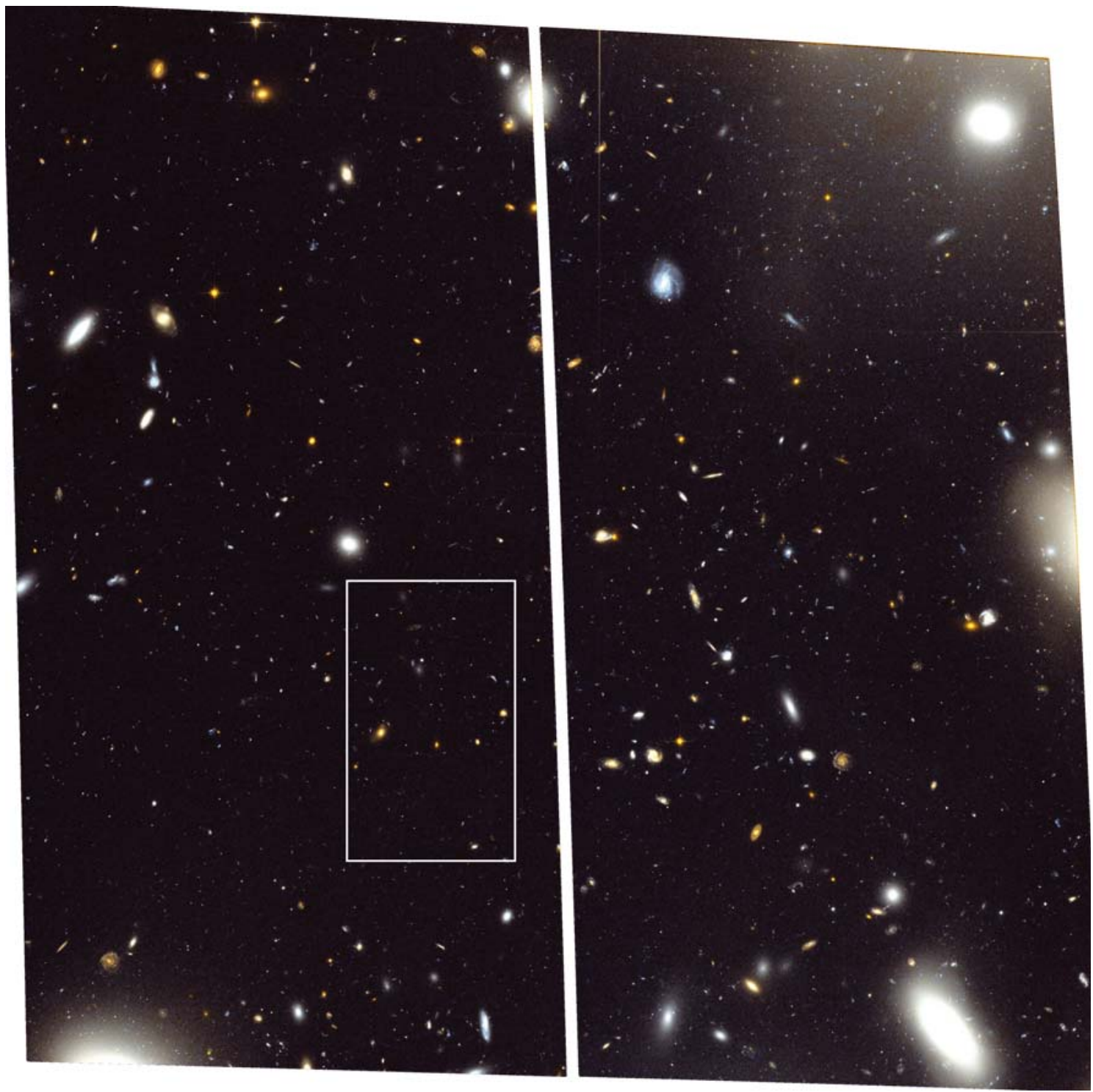

Fig. 2. ACS image of the central field in Abell 1185, produced using the $V_{F 555 W}$ and $I_{F 814 W}$ images and averaging them to produce the third color. The brightest cluster galaxy, NGC 3550, is located just off the upper right corner of the image. The field of view is $3.4^{\prime} \times 3.4^{\prime}$, corresponding to a spatial scale of $\sim 220 \mathrm{kpc}$ on a side. North is up and east is to the left. The white box indicates the region shown in Fig. 5.

for typical globular cluster colors $(V-I \simeq 1)$. The relatively shallower limit of the $V_{F 555 W}$ images was intended to provide color information for brighter globular clusters only. With a scale of $0.049^{\prime \prime}$ per pixel, the images have a $202^{\prime \prime} \times 202^{\prime \prime}$ field of view corresponding to $\sim 130 \mathrm{kpc}$ on a side at the distance of A1185 (for an assumed distance modulus $m-M=35.7$ ). Individual exposures were not dithered because the small gap between the ACS chips does not adversely affect our ability to detect globular clusters over most of the field.

Images were processed using the Apsis ACS data pipeline (Blakeslee 2003), which produces final sky-subtracted images in each filter after distortion correction, image alignment, cosmic ray removal and image combination using sub-pixel drizzling (Fruchter \& Hook 2002) with a final pixel scale of $0.05^{\prime \prime}$. A combined $V_{F 555 W}+I_{F 814 W}$ image is shown in Fig. 2. Weight images were also constructed for each output science image; these give the total uncertainty for each pixel based on instrumental and photon shot noise (see Jordán et al. 2004). These weight images provide a map of the rms variation in the final image that was used for object detection as described below.

\subsection{Object detection and classification}

Object detection was performed using the Source Extractor (SExtractor) software package (Bertin \& Arnouts 1996) to produce a catalog of candidate globular clusters. At the distance of A1185, globular clusters are unresolved even with ACS and hence will appear as faint point sources (a typical globular cluster with half-light radius $r_{\mathrm{h}} \simeq 3 \mathrm{pc}$ would have an angular size much less than one ACS/WFC pixel, however this is diffused over several pixels because of the ACS PSF which has a $F W H M \sim 2$ pixels).

Objects were selected using a detection threshold of five contiguous pixels each at least $1.5 \sigma$ above background after convolving the image with a Gaussian filter gauss_2. $\theta_{-} 5 \times 5$. The rms maps produced by Apsis were used to weight each pixel based on its noise level by setting the WEIGHT_TYPE parameter to MAP_RMS when running SExtractor. The local background was estimated using a mesh size of $40 \times 40$ square pixels. These detection parameters were chosen after experimentation as providing the best compromise between sensitivity 


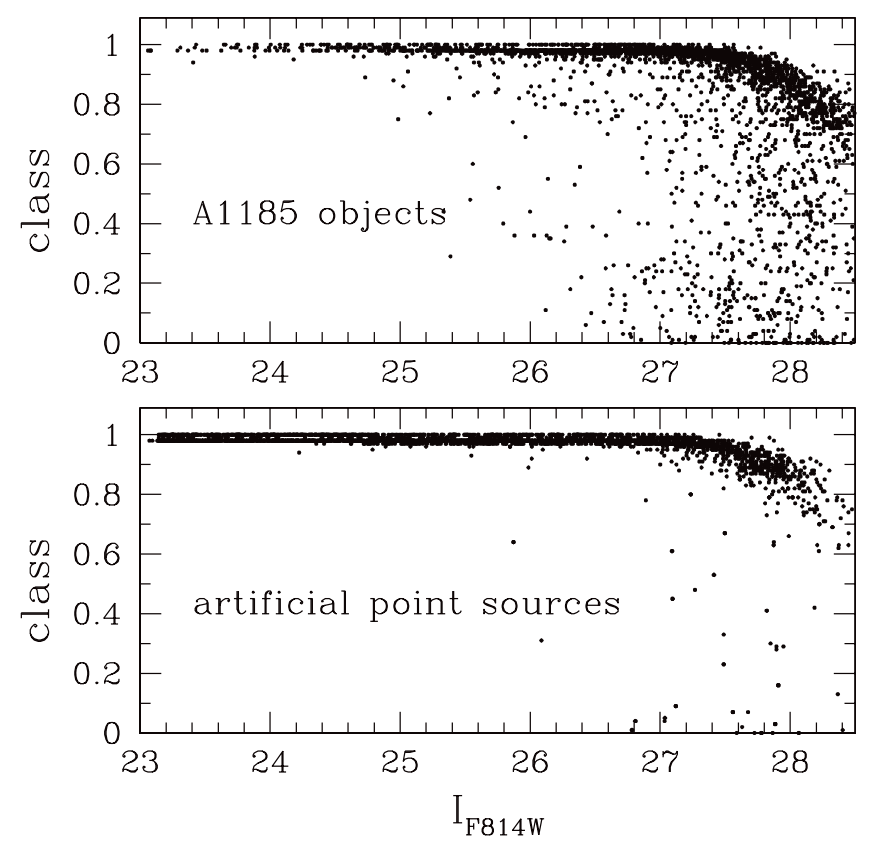

Fig. 3. Top: SExtractor classes of all detected objects in the core of A1185. Bottom: SExtractor classes of artificial point sources that were added to the field as described in Sect. 2.3. Our adopted criterion of CLASS $\geq 0.8$ for globular cluster candidates does an excellent job of identifying point sources down to the faintest magnitudes.

and reliability. Object detection was done independently for the $F 555 W$ and $F 814 W$ images and the resulting object catalogs were then matched using a $0.1^{\prime \prime}$ matching radius.

SExtractor assigns each detected object a classification parameter, CLASS, that ranges from $\sim 0$ for galaxies and other extended objects to $\sim 1$ for point sources. CLASS values are based on a neural network algorithm trained on simulated images (for details see Bertin \& Arnouts 1996). We consider point source candidates to have CLASS $\geq 0.8$. Figure 3 shows that this criterion does an excellent job of discriminating globular clusters from galaxies and other non-point sources even at the faintest magnitudes. Experiments in which artificial point sources of different magnitudes were added at random locations in the ACS field (see Sect. 2.3 for details) show that fully $98 \%$ of all input objects detected by SExtractor down to $I_{F 814 W}=28$ mag were assigned CLASS $\geq 0.8$.

\subsection{Photometric calibration}

Because globular clusters are unresolved at the distance of A1185, we used the ALLSTAR routine in the DAOPHOT software package (Stetson 1987) to perform photometry of detected candidates based on PSF fitting, which provides more reliable magnitudes than the default SExtractor magnitudes.

To do this, the coordinates of SExtractor-detected objects were fed into DAOPHOT/ALLSTAR and instrumental magnitudes were then measured at each location using PSF models determined empirically from stars on the final $F 555 \mathrm{~W}$ and $F 814 \mathrm{~W}$ images. We followed the procedures of Sirianni et al. (2005) for aperture corrections and converted instrumental magnitudes to the VEGAMAG system using the following zeropoints:

$$
\begin{gathered}
V_{555 W}=-2.5 \log f_{555 W}+25.724 \\
I_{814 W}=-2.5 \log f_{814 W}+25.501
\end{gathered}
$$

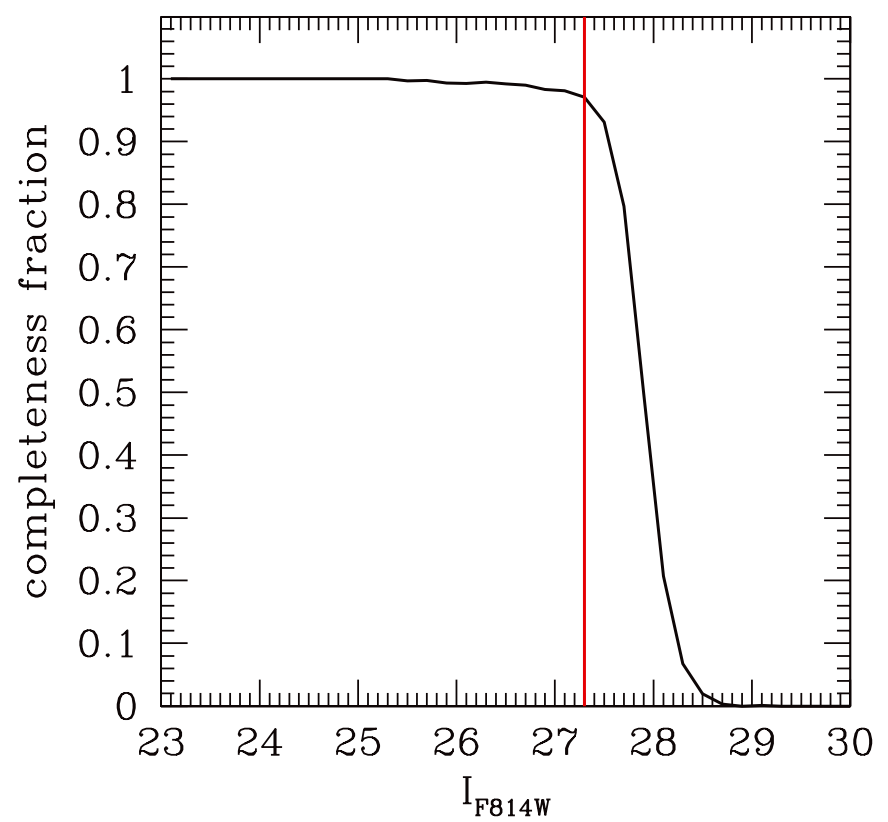

Fig. 4. Completeness of point source detection as a function of apparent magnitude for the $F 814 \mathrm{~W}$ image of the central region in A1185. The red line shows the expected turnover magnitude of the globular cluster luminosity function at $I_{F 814 \mathrm{~W}} \simeq 27.3 \mathrm{mag}$.

where $f_{555 \mathrm{~W}}$ and $f_{814 \mathrm{~W}}$ are the integrated fluxes in units of electrons per second in each filter. Reddening for the A1185 field was taken to be $E(B-V)=0.029$ mag based on the DIRBE maps of Schlegel et al. (1998) and extinction ratios were taken from Sirianni et al. (2005).

\subsection{Completeness limits}

Object detection and classification become increasingly unreliable at faint magnitudes. To assess our completeness limits, artificial stars of known magnitudes were added at random locations in the final ACS images and then the same SExtractor and DAOPHOT procedures described above were used to determine the fraction of stars recovered at each magnitude. These experiments were performed by adding 100 stars at random to the ACS field using the DAOPHOT task ADDSTAR. SExtractor was then run with the same detection parameters and the fraction of input stars that were recovered was recorded. This process was repeated many times to generate the completeness function shown in Fig. 4 for the $F 814 W$ observations.

These experiments show that object detection and classification are essentially $100 \%$ complete down to the expected turnover magnitude of the GCLF at $I_{F 814 W} \simeq 27.3 \mathrm{mag}$, and declines rapidly at fainter magnitudes. To avoid uncertainties caused by incompleteness at fainter magnitudes, we adopted a completeness limit at $I_{F 814 W} \simeq 27.3$ mag and consider only objects brighter than this in subsequent analysis.

\section{Results}

Globular clusters detected in a small portion of the A1185 core field are shown in Fig. 5. A total of 2285 such objects selected solely on the basis of their SExtractor classifications were found in the $F 814 \mathrm{~W}$ image, of which 1422 are brighter than our adopted completeness limit $I_{F 814 W}=27.3 \mathrm{mag}$. The projected 


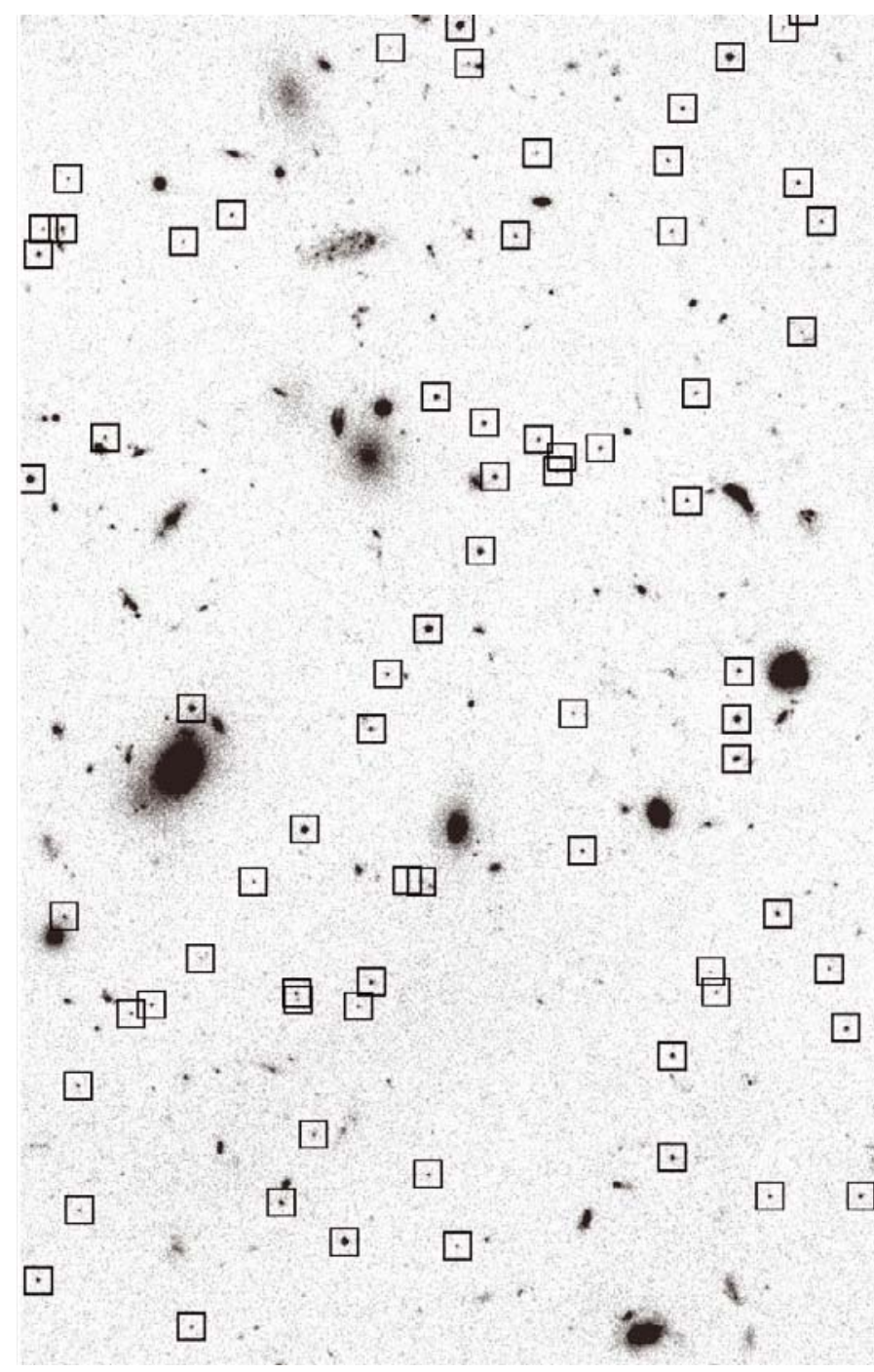

Fig. 5. Intergalactic globular cluster candidates (in boxes) found in a small portion of the ACS field in the core of Abell 1185. The field of view is $\sim 0.3^{\prime} \times 0.5^{\prime}$.

spatial distribution of such objects is plotted in Fig. 6. Matched detections of 1124 objects were found in the $F 555 \mathrm{~W}$ image.

It is clear from Fig. 6 that the two-dimensional distribution of IGC candidates is not uniform. A prominent enhancement in the direction of NGC 3550 is easily seen, as are several other concentrations (e.g., around $x \simeq 500, y \simeq 300$ associated with the galaxy MCG+05-27-004 that lies just outside the ACS field). Hence we are undoubtedly seeing some contamination from globular clusters in the halos of galaxies, which we address below.

\subsection{The number of IGCs in $A 1185$}

Jordán et al. (2003) detected 99 IGC candidates in A1185 based on shallower WFPC2 observations. If intergalactic globular clusters have the same luminosity function as their counterparts in galaxies, then scaling from the WFPC2 results to the depth and area of our ACS field (assuming a constant density of objects across the field) we would expect to find approximately 2000 to 4000 globular clusters in this region, with about half this number detected down to our adopted $100 \%$ completeness limit at

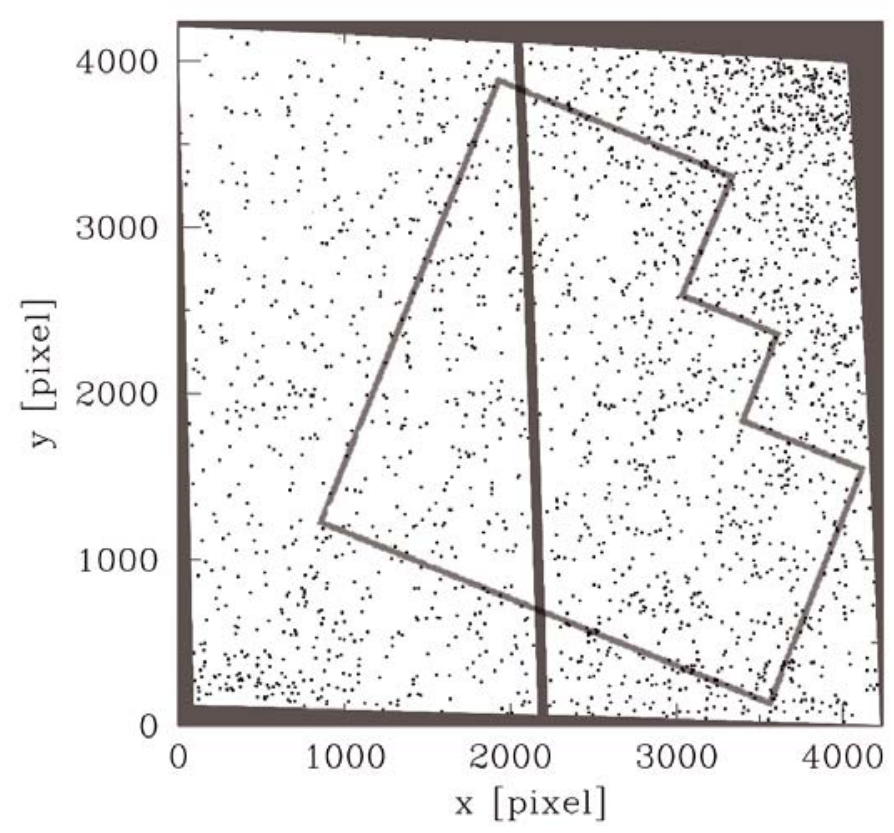

Fig. 6. The distribution of the 1422 intergalactic globular cluster candidates brighter than $I_{F 814 W}=27.3 \mathrm{mag}$ in the A1185 core field. The field of view is $3.4^{\prime} \times 3.4^{\prime}$, with north up and east to the left. Enhancements in the number of objects can be seen in a few regions, especially in the direction of the bright galaxy NGC 3550. For comparison, the location of the smaller and shallower WFPC2 field studied by Jordán et al. (2003) is also shown.

$I_{F 814 W} \simeq 27.3 \mathrm{mag}$. The detection of 1422 objects brighter than this magnitude limit is consistent with this estimate.

However, it is inevitable that some IGC candidates are actually foreground stars, unresolved background galaxies, and globular clusters in the halos of galaxies near the ACS field masquerading as IGCs. Confirming the presence of IGCs in A1185 and estimating their number requires correcting for these various sources of contamination.

To assess the contribution from stars and unresolved galaxies along the line of sight, four fields observed with the same instrument, filter and comparable integration times were processed and analyzed in an identical manner as the A1185 core field. The selected fields, which are all at similar Galactic latitudes as the A1185 field, are devoid of bright galaxies and can therefore provide unbiased estimates of the number of contaminating point sources at different magnitudes. The four fields used were:

- a second field in A1185 (see Fig. 1) was observed as part of our programme GO-10277. This field is located $\sim 350 \mathrm{kpc}$ from the primary field and hence can provide a useful constraint on the spatial extent of the IGC distribution within A1185. ACS observations with integration times of 17816 seconds were obtained for both the $F 555 \mathrm{~W}$ and F814W filters;

- F814W images of a field observed to study high-redshift Ly- $\alpha$ emitters (GO-9411) were extracted from the HST archive. This field is at Galactic latitude $b=68^{\circ}$, almost identical to that of A1185. A subset of 16 images were combined to attain a total exposure time of $24060 \mathrm{~s}$;

- two additional fields from the Gemini Deep Deep Survey (GO-9760) were obtained from the HST archive. These are the SA02-1 and SA02-2 fields, which are located well away 


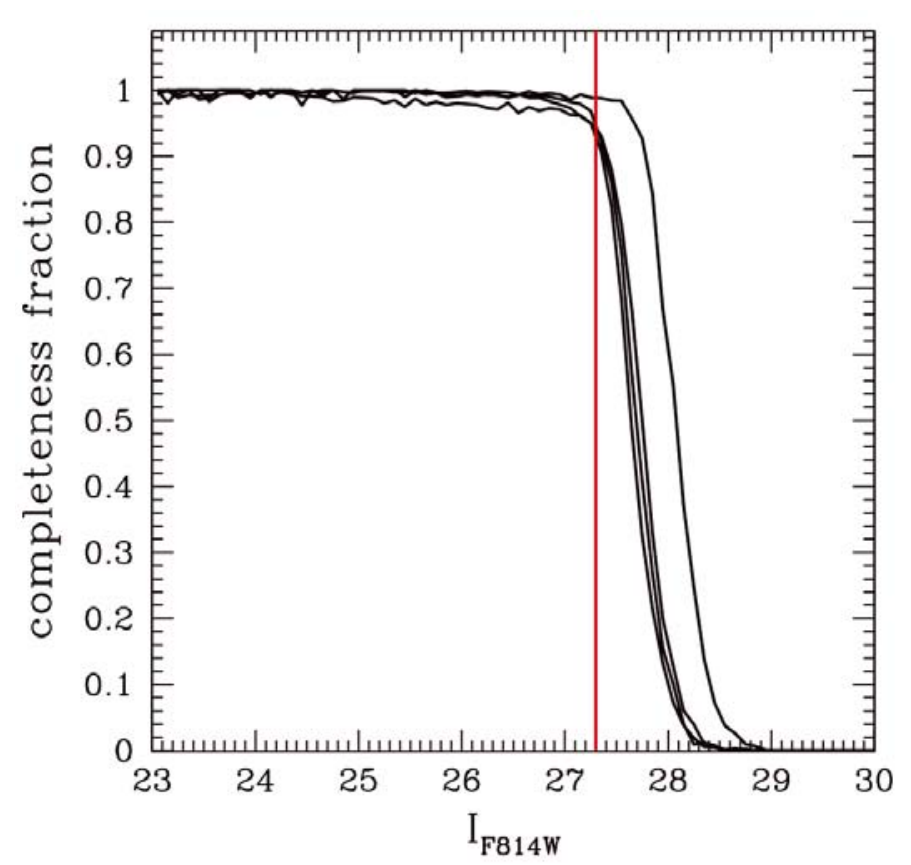

Fig. 7. Completeness of point source detection as a function of $I_{F 814 W}$ magnitude for the four control fields. The red line shows the expected turnover magnitude of the globular cluster luminosity function at $I_{F 814 W} \simeq 27.3$ mag. Detection of point sources is nearly $100 \%$ complete down to this magnitude in all four control fields. The completeness fraction at faint magnitudes is greatest for the GO-9411 field, which has the longest total integration time.

from the Galactic plane at $b=-61^{\circ}$. The total integration time for both fields was $16345 \mathrm{~s}$ with the $F 814 W$ filter.

Figure 7 shows the completeness functions for each of the control fields determined from artificial star experiments. All four show similar completeness limits, with nearly $100 \%$ of objects detected down to $I_{F 814 W} \simeq 27.3 \mathrm{mag}$, which is not surprising given their similar integration times.

In Fig. 8 we plot the number of detected point sources as a function of $I_{F 814 W}$ magnitude for all fields. The agreement between the four control fields is remarkably good, which suggests that they provide a reliable estimate of the number of contaminating foreground and background point sources along the line of sight to A1185. Furthermore, the fact that the counts in the second (outer) A1185 field are statistically indistinguishable from those in the three other control fields indicates that globular clusters are not present in significant numbers in this region, and hence if an IGC population is present in A1185 then it must be concentrated towards the innermost regions.

Averaging results from the four control fields, we conclude that the number of contaminating foreground stars and background galaxies is $\simeq 142 \pm 12$ down to a magnitude $I_{F 814 W}=$ $27.3 \mathrm{mag}$. The number of objects detected in the A1185 core field is an order of magnitude greater than this, a total of $\sim 1300$ globular cluster candidates. Likewise, Fig. 9 shows the color-magnitude relation for point sources in the A1185 core field compared with that of similar objects in the outer field of A1185. If we consider only those objects with colors in the range $0.7 \leq V-I \leq 1.5$ expected for globular clusters, then there are 977 objects in the central field compared to 32 in the outer field, strong evidence of the presence of a large population of globular clusters in the core of A1185. Based on our detection of 1422 globular cluster candidates brighter than

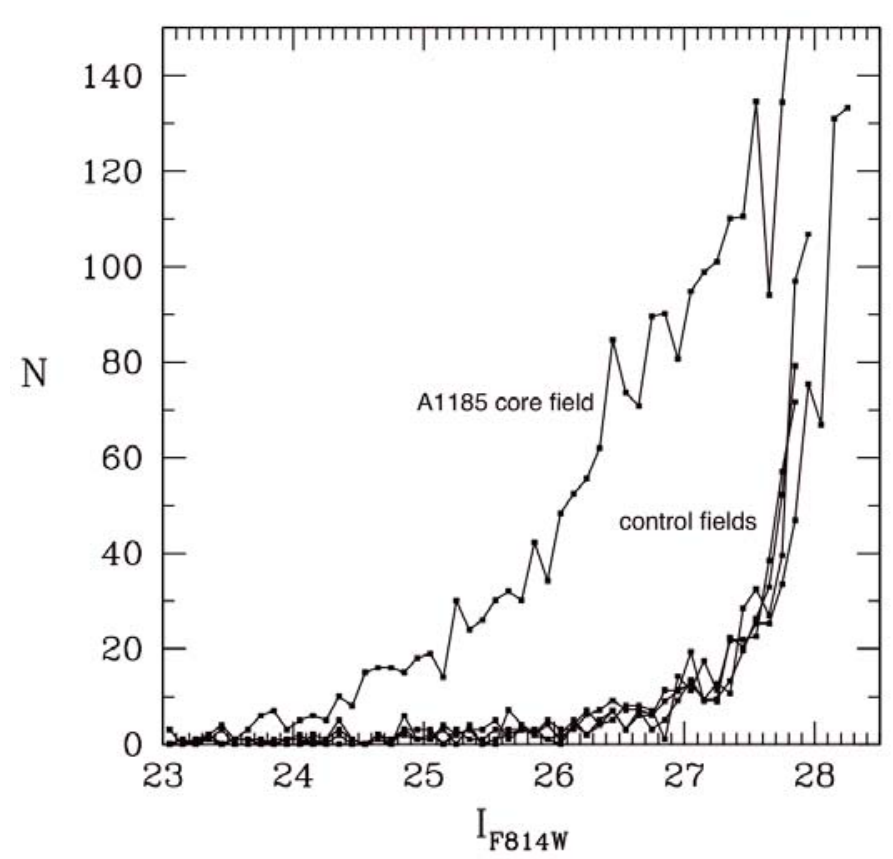

Fig. 8. The number of detected point sources in the A1185 core field and the four control fields. Counts have been corrected for incompleteness at each magnitude. A large excess of objects is clearly seen at all magnitudes in the core field compared to the control fields, consistent with the presence of a substantial population of globular clusters.

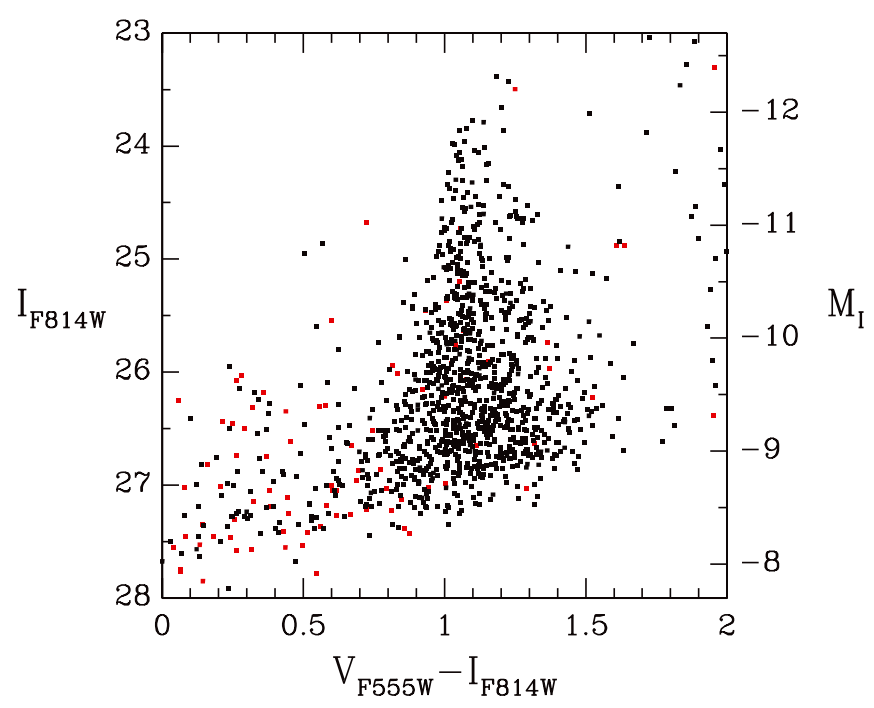

Fig. 9. Color-magnitude diagram for globular-cluster-like objects in A1185 core field. Black points are objects in the core field and red points are from the outer field indicated in Fig. 1. The prominent swath of objects with colors $0.7 \leq V_{F 555 W}-I_{F 814 W} \leq 1.5$ shows the detection of a substantial number of globular clusters in the core field. Within this color range there are 977 objects in the central field compared to only 32 in the outer field, a factor of more than 30 enhancement in the number of objects. $M_{I}$ denotes the absolute magnitude of detected objects assuming a distance modulus $m-M=35.7$.

$I_{F 814 \mathrm{~W}}=27.3 \mathrm{mag}$, after subtracting the expected number of contaminating background objects and doubling the resulting number to include the faint half of the GCLF, we estimate that $\sim 2560$ globular clusters in total are present in the ACS field.

To estimate the fraction of the detected globular clusters that are associated with galaxies, we performed detailed simulations 
Table 1. Galaxies near the center of A1185 used to estimate contamination in the ACS field from halo globular clusters.

\begin{tabular}{lcccc}
\hline \hline Galaxy & RA (2000) & Dec (2000) & $V(\mathrm{mag})$ & $z$ \\
\hline NGC 3550 & $11: 10: 38.7$ & $28: 46: 06$ & 13.2 & 0.0346 \\
NGC 3552 & $11: 10: 42.8$ & $28: 41: 35$ & 14.3 & 0.0331 \\
CGCG 155-081 & $11: 10: 37.5$ & $28: 43: 58$ & 14.9 & 0.0310 \\
MCG+05-27-004 & $11: 10: 52.0$ & $28: 42: 09$ & 15.3 & 0.0340 \\
NGC 3553 & $11: 10: 40.5$ & $28: 41: 05$ & 15.3 & 0.0326 \\
2MASX J11105941+2844424 & $11: 10: 59.4$ & $28: 44: 43$ & 15.7 & 0.0327 \\
2MASX J11104649+2841338 & $11: 10: 46.5$ & $28: 41: 34$ & 15.9 & 0.0326 \\
2MASX J11103987+2842248 & $11: 10: 39.9$ & $28: 42: 25$ & 16.3 & 0.0288 \\
2MASX J11103964+2845208 & $11: 10: 39.6$ & $28: 45: 21$ & 16.9 & 0.0383 \\
2MASX J11103683+2842138 & $11: 10: 36.8$ & $28: 42: 14$ & 16.9 & - \\
2MASX J11104185+2845408 & $11: 10: 41.9$ & $28: 45: 41$ & 17.0 & - \\
2MASX J11105850+2842384 & $11: 10: 58.5$ & $28: 42: 39$ & 17.1 & 0.0346 \\
SDSS J111035.04+284205.2 & $11: 10: 35.0$ & $28: 42: 05$ & 17.3 & 0.0338 \\
2MASX J11103767+2841528 & $11: 10: 37.7$ & $28: 41: 53$ & 17.6 & 0.0343 \\
\hline
\end{tabular}

of the expected distribution of globular clusters belonging to all galaxies brighter than $V \simeq 18\left(M_{V} \simeq-18\right)$ within $3^{\prime}$ of the centre of the A1185 core field (fainter or more remote galaxies are unlikely to contribute contaminating globular clusters in significant numbers). These 14 galaxies and their properties are listed in Table 1 along with their apparent $V$-band magnitudes measured from the CFH12k images of Andreon et al. (2007) using SExtractor and their redshifts when known. Although two of these galaxies do not have measured redshifts, we have assumed that all are members of A1185. The following procedures and input parameters were used to generate the simulations:

- It is well established that the number of globular clusters belonging to a galaxy scales with its luminosity. A standard measure is the specific globular cluster frequency, $S_{N}$, defined as

$S_{N}=N_{\mathrm{gc}} 10^{-0.4\left(M_{V}+15\right)}$

where $N_{\mathrm{gc}}$ is the total number of globular clusters and $M_{V}$ is the galaxy's absolute $V$-band magnitude (Harris \& van den Bergh 1981). Blakeslee et al. (1997) measured the specific frequency of the dominant elliptical, NGC 3550, to be $S_{N} \simeq 6.5$, which is in the normal range for a galaxy of its luminosity. For the other galaxies, none of whose globular cluster systems have been studied to date, we used the empirical relation between specific frequency and $V$-band galaxy luminosity determined by Peng et al. (2008) from observations of 100 early-type galaxies in the ACS Virgo Cluster Survey (Côté et al. 2003), with $S_{N}$ values ranging from $\simeq 1-5$ and the $V$-band magnitudes measured directly from the CFH12k images of Andreon et al. (2007) using SExtractor. This relation agrees well with that found for other galaxies (see, e.g., Ashman \& Zepf 1998, and references therein) and hence provides a reliable input for the simulations. A total number of globular clusters was assigned to each galaxy in Table 1 based on its luminosity and assumed specific frequency.

- Each simulated globular cluster was assigned a luminosity by randomly sampling a Gaussian luminosity function with a mean absolute magnitude of $M_{I}=-8.4 \mathrm{mag}$ and a standard deviation $\sigma=1.4 \mathrm{mag}$, which is also consistent with previous observations of the GC population of NGC 3550 (Blakeslee et al. 1997). Clusters whose apparent magnitude at the distance of A1185 would be fainter than our $I_{F 814 \mathrm{~W}}=27.3 \mathrm{mag}$ completeness limit were then removed from further consideration. Although Jordán et al. (2007) and
Villegas et al. (2010) have shown that the properties of the GCLF correlate with parent galaxy luminosity, it is nearly uniform among high-mass galaxies that contribute the bulk of contaminating globular clusters, with turnover magnitudes and dispersions in agreement with the values adopted here. The five most luminous galaxies in Table 1, for example, which contribute the lion's share of halo globular clusters, would be expected to have $\sigma$ ranging from $\sim 1.2$ to $1.4 \mathrm{mag}$. Hence, any non-universality of the GCLF can safely be ignored in the present analysis.

- The spatial distribution of globular clusters in galaxy halos varies widely but is generally more extended than that of the starlight (e.g., Harris 1991; Ashman \& Zepf 1998; Brodie \& Strader 2006). We assumed that the globular cluster system around each galaxy has a spherical distribution that follows a Sérsic (1968) profile

$I(r)=I_{\mathrm{e}} \exp \left[-b_{n}\left(r / r_{\mathrm{e}}\right)^{1 / n}-1\right]$

where $I_{\mathrm{e}}$ is the projected surface brightness at the effective radius, $r_{\mathrm{e}}$, that contains half the total light of the system, $n$ is the Sérsic index that determines the overall shape of the profile, and $b_{n}$ is a parameter related to $n$ (see Graham \& Driver 2005, and references therein). Surface brightness profiles for the stellar distribution in each galaxy were measured on the CFH12k R-band image of A1185 (Fig. 1) using the ellipse routine in IRAF, and Sérsic parameters were then obtained by fitting the profiles to Eq. (3) using $\chi^{2}$ minimization $^{1}$. As an initial guess, the simulations assumed that the effective radius of each galaxy's globular cluster distribution is twice that of its stellar distribution (Peng et al. 2011).

Tidal forces from the gravitational field of A1185 and galaxy-galaxy interactions are likely to limit the radial extent of each galaxy's globular cluster distribution (e.g., Merritt 1984; although see Mihos et al. 2005; and Janowiecki et al. 2010). For this reason we simulated three different scenarios. For one, the globular cluster distribution was not truncated, which likely leads to an overestimate of the amount of contamination from halo globular clusters in our ACS field. For the other cases, the globular cluster population of each

1 As Fig. 10 shows, NGC 3550 is actually a merging system of three galaxies within a common envelope, a brightest cluster galaxy caught in the act of formation. The light from the two companion galaxies was subtracted before measuring the underlying surface brightness profile of NGC 3550 . 

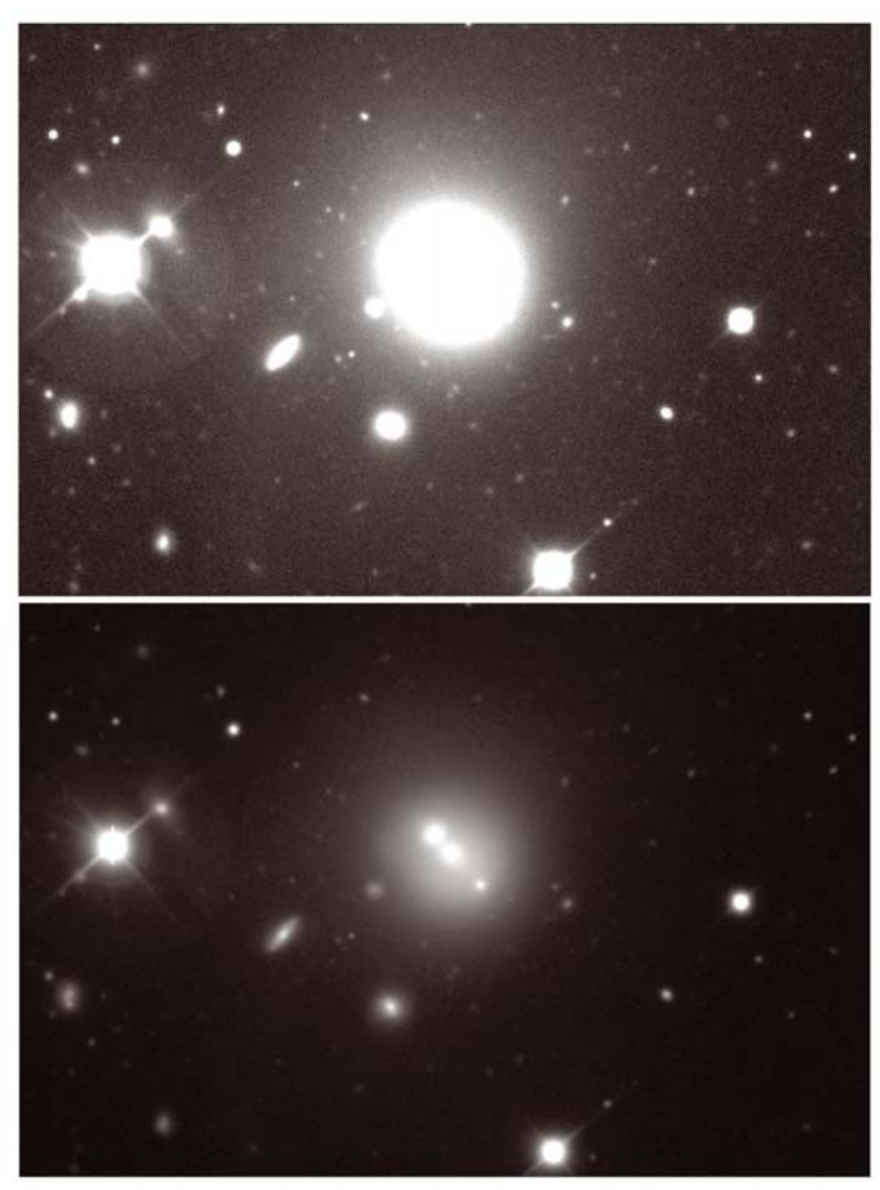

Fig. 10. NGC 3550, the brightest member galaxy in A1185. The image on top is displayed with a linear intensity scale and NGC 3550 appears as a single large elliptical galaxy. The logarithmic display below reveals that NGC 3550 is actually a complex system of three smaller galaxies within a common envelope.

galaxy was truncated beyond a radius of $50 \mathrm{kpc}$ or $100 \mathrm{kpc}$. Previous observations by Blakeslee (1996) showed that the number density of point sources surrounding NGC 3550 becomes constant beyond a projected distance of $100^{\prime \prime}$, suggesting that this galaxy's globular cluster population does not extend beyond $\sim 63 \mathrm{kpc}$. Similarly, in a wide-field imaging survey Rhode \& Zepf $(2001,2004)$ found that the maximum radial extent of the globular cluster systems of four earlytype galaxies in the Virgo cluster ranged from $\sim 30-100 \mathrm{kpc}$. The assumed radial distribution of globular clusters in galaxy halos is the greatest uncertainty in these models.

Using the procedures and input parameters described above, 100 random realizations of the expected distribution of globular clusters in the halos of the galaxies in Table 1 were generated and the simulated clusters brighter than $I_{F 814 W} \leq 27.3$ mag that fall within the ACS field boundaries were counted. An example is shown in Fig. 11.

Based on these simulations, we estimate that $\sim 711 \pm 26$ IGC candidates in the A1185 core field are likely to be globular clusters in galaxy halos if their spatial extent in unlimited, most of them belonging to NGC 3550 . We emphasize, however, that this probably overestimates the number of contaminating halo globular clusters. For the case in which each galaxy's globular cluster system is assumed to be tidally truncated beyond $100 \mathrm{kpc}$, the estimated number of contaminating objects is $\sim 472 \pm 30$, and for a limiting radius of $50 \mathrm{kpc}$ the number falls to $\sim 166 \pm 14$.

Combining the estimated contamination from globular clusters in galaxy halos and the expected number of unassociated objects along the line of sight, we estimate that less than half of the 1422 IGC candidates brighter than $I_{F 814 W} \simeq 27.3$ mag can be accounted for this way, and hence the majority of detected objects are likely to be genuine IGCs. Our best estimate, based on the assumption that globular clusters are not found beyond $100 \mathrm{kpc}$ in galaxy halos, is that $\sim 1616 \pm 91$ IGCs of all magnitudes exist in the core ACS field ${ }^{2}$.

However, although there is no doubt that we have detected a large population of globular clusters in the core of A1185, it is difficult to say with certainty whether any of them are truly intergalactic. In addition to the aforementioned assumptions and uncertainties inherent in our simulations, other factors might also plausibly raise the number of globular clusters associated with galaxies. For example, although low- or intermediate-luminosity galaxies (fainter than $M_{V} \simeq-18$ ) have not been included in the simulations because their globular cluster populations are expected to be sparse, a large population of such galaxies could collectively contribute several hundred additional contaminating halo clusters scattered over the ACS field. Uncertainties in the magnitudes of the galaxies in Table 1 could also raise or lower the expected number of galactic globular clusters.

It is, of course, possible to boost the predicted number of contaminating objects to match the number of detected objects in the ACS field by arbitrarily increasing the globular cluster populations of the galaxies or changing their radial distributions. For example, doubling the assumed specific frequency of every galaxy in Table 1 and assuming that assuming that the globular cluster distribution is twice as extended as the galaxy light yields an estimated $1292 \pm 34$ contaminating halo globular clusters in the ACS field. However, such a model seems unlikely, especially in light of the measured specific frequency of NGC 3550 (Blakeslee et al. 1997). To further assess the validity of such a high specific frequency model, the simulated distributions of globular clusters (including the addition of $142 \pm 12$ randomly distributed foreground/background objects) were compared to the observed distribution using a two-dimensional KolmogorovSmirnov test (Peacock 1983; Fasano \& Franceschini 1987) as implemented by Press et al. (1992). Based on this analysis, the probability that the simulated and observed globular cluster distributions could both be drawn from the same parent distribution is much less than $1 \%$, although one must bear in mind the limitations of these models.

Given the uncertainties in the simulations, and to explore the full range of parameter space - including more extreme possibilities - several thousand additional simulations were run in which the parameters of each galaxy's globular cluster system were randomly chosen in the range $1 \leq S_{N} \leq 20,0^{\prime \prime} \leq r_{\mathrm{e}} \leq 200^{\prime \prime}$ and $0 \leq n \leq 10$. These experiments demonstrate that, even in the most favorable case in which the spatial extent of each galaxy's GC system is not tidally limited, a minimum necessary condition

2 This estimate of $\sim 1616$ IGCs is based on the detection of 1422 objects down to $I_{F 814 W} \simeq 27.3 \mathrm{mag}$, subtracting 142 background objects brighter than this same limiting magnitude and assuming that 472 of the IGC candidates are globular clusters in galaxy halos. The resulting number is then doubled to account for the fact that we observe only the brighter half of the GCLF. Formal statistical uncertainties in the number of detected IGCs, unrelated point sources, and halo globular clusters have been added in quadrature, however systematic errors resulting from our lack of knowledge about the globular cluster systems of individual galaxies make the results considerably more uncertain. 

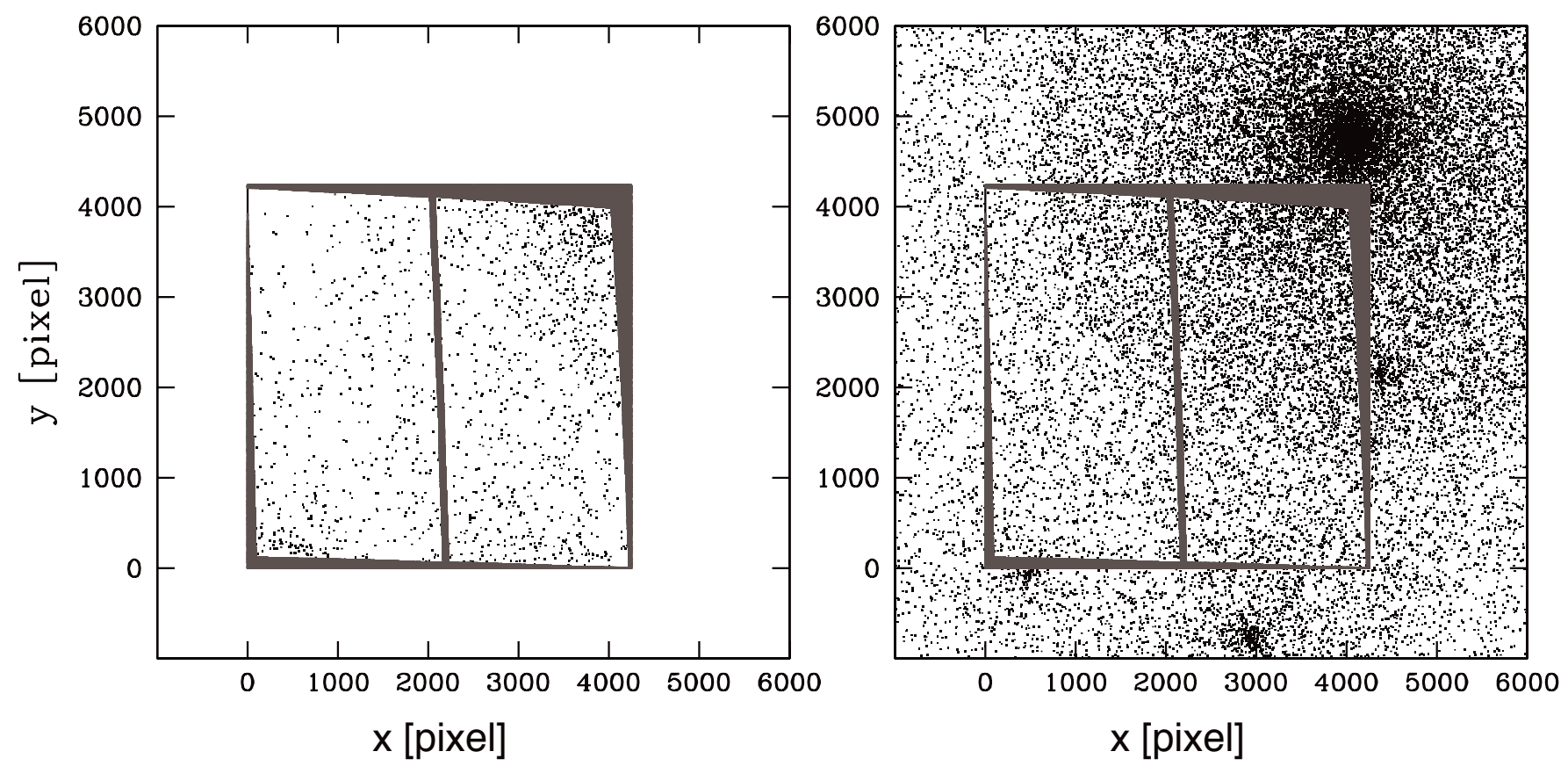

Fig. 11. Simulations of the expected contamination from globular clusters in halos of galaxies that lie near the A1185 core field. The left panels shows the real distribution of globular clusters brighter than $I_{F 814 W}=27.3$ mag in the ACS image of the heart of 1185. The right panel shows a superposition of 10 simulated distributions of halo globular clusters associated with the galaxies listed in Table 1 down to the same $I_{F 814 W}=$ 27.3 mag limit and assuming no truncation of their radial distribution, as described in the text.

for $~ 1000-1500$ contaminating halo globular clusters to fall within our central ACS field is that two or more of the brightest member galaxies (NGC 3550, NGC 3552, CGCG 155-081 and MCG +05-27-004) would have to possess extremely rich globular cluster systems, with $S_{N} \geq 10-20$ (see Fig. 12 for an illustration). While this possibility cannot be ruled out, it seems unlikely that A1185 would have the distinction of being the only known cluster with multiple high $S_{N}$ galaxies, especially given the unexceptional size of NGC 3550's globular cluster population (Blakeslee et al. 1997).

We conclude that, for any reasonable assumptions, it is difficult to account for the large number of globular clusters detected in our central ACS field without including a significant population of IGCs that do not belong to galaxies. Although we cannot completely rule out the possibility that most or all of the globular clusters detected in the core of A1185 could be associated with galaxies, this seems unlikely. Further insights into the nature of these objects could come from their luminosities and colors, which are examined in more detail below.

\subsection{The globular cluster luminosity function}

The luminosity function (or, equivalently, the mass function) is a fundamental property of globular cluster systems. The presentday GCLF has been shaped by many factors, including physical conditions at the time of their formation, internal processes such as stellar mass loss and two-body relaxation, and stripping or destruction of clusters in galaxy environments as a result of disk shocking, tidal forces, and dynamical friction. Given this variety of influences, it is remarkable that the GCLF in galaxies can, to first order, be described universally by a Gaussian distribution with a maximum at $M_{I} \simeq-8.4$ and a dispersion of $\sigma \simeq$ 1.2-1.4 mag (e.g., Harris 1991). However, some correlation is observed between GCLF properties and parent galaxy luminosity (Jordán et al. 2006, 2007; Villegas et al. 2010), which suggests that environment plays a role in the formation and/or evolution of globular cluster systems. Several authors have shown, for example, that an initial power-law mass function can be transformed into one with a log-normal shape as a consequence of cluster disruption and evaporation in galaxies (see, e.g., Fall \& Zhang 2001; Vesperini et al. 2003; McLaughlin \& Fall 2008; Elmegreen 2010).

It therefore seems plausible that the luminosity function of IGCs born outside of galaxies might differ from that of globular clusters which formed and evolved within galaxies, although it is hard to predict a priori what form such differences might take. If, on the other hand, IGCs originated in galaxies and were subsequently stripped from halos or spilled into intergalactic space during the disruption of their parent galaxies then the IGC luminosity function is likely to be similar to that of galactic globular clusters. Hence the luminosity function of globular clusters in the core of A1185 might provide some insights into their origin.

Figure 13 shows the observed luminosity function of globular cluster candidates based on the deep $F 814 \mathrm{~W}$ observations. The number of objects in each luminosity bin has been corrected by subtracting the expected contamination from foreground stars and unresolved background galaxies (Fig. 8) and correcting for incompleteness (Fig. 4). Using $\chi^{2}$ minimization to fit the observed GCLF to a Gaussian distribution with the turnover magnitude fixed at $I_{F 814 W}=27.3$ mag but with the amplitude and width of the distribution as free parameters yields a best fit with $\sigma \simeq 1.2 \pm 0.2$, which is shown for comparison in the figure. Within the uncertainties, this falls well within the normal range for GCLFs in galaxies. 


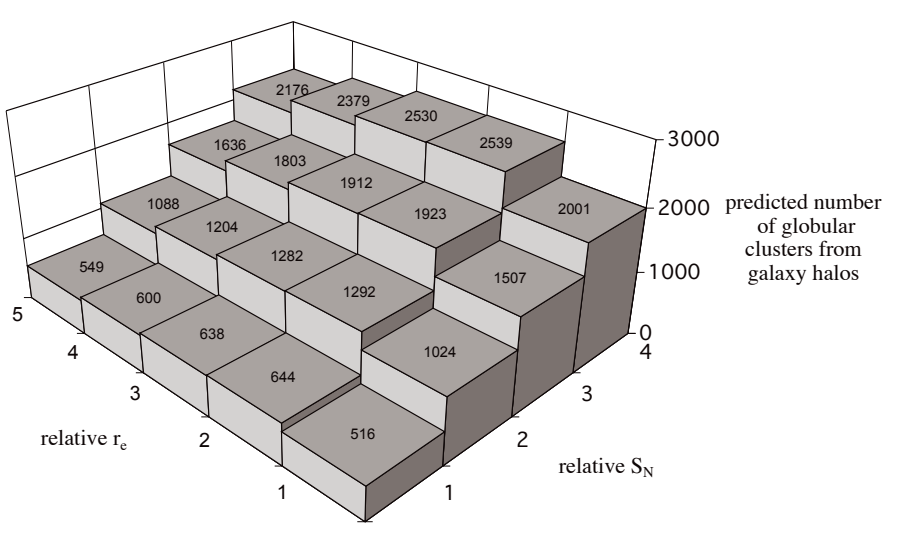

Fig. 12. An illustration of the expected contamination from globular clusters in galaxy halos near the center of A1185 and its dependence on the assumed properties of those systems. The globular cluster population of each galaxy in Table 1 was simulated by assuming that its radial distribution is described by the same Sersic index $n$ as the galaxy's stellar light, but with an effective radius that is some multiple of the galaxy's (the axis labelled "relative $r_{\mathrm{e}}$ "). Similarly, the number of globular clusters belonging to each galaxy was allowed to vary relative to the expected number if the galaxy had a normal specific frequency for its luminosity as summarized in Peng et al. (2008) (the axis labelled "relative $S_{N}$ "). Hence, for example, a relative $r_{\mathrm{e}}=2$ means that the effective radius of a galaxy's globular cluster distribution was assumed to be twice that of its stellar distribution, and an "effective $S_{N}=3$ " means that a galaxy was assigned three times the "normal" number of globular clusters for a galaxy of its luminosity. Additional details of the simulations are discussed in the text. The third axis shows the average number of contaminating globular clusters brighter than $I_{F 814 W} \leq 27.3$ mag predicted to fall within the central ACS field in A1185 based on many simulations. Although the relative $r_{\mathrm{e}}$ and $S_{N}$ were allowed to vary independently for each galaxy, for clarity the results shown in this figure assume constant values of relative $r_{\mathrm{e}}$ and relative $S_{N}$ for all galaxies. These simulations demonstrate that the large number of globular clusters seen in our ACS field cannot all reside in galaxy halos unless most or all of the galaxies in Table 1 have unusually rich globular cluster systems.

\subsection{The globular cluster color distribution}

It is well established that the colors of old globular clusters, which are determined primarily by their metallicities, show a dependence on parent galaxy luminosity, in the sense that luminous galaxies have redder (more metal-rich) globular clusters on average than fainter galaxies (e.g., van den Bergh 1975; Brodie \& Huchra 1991; Forbes \& Forte 2001; Côté et al. 1998; Peng et al. 2006). Hence the color distribution of globular clusters in the core of A1185 could, in principle, provide some constraints on the numbers and types of donor galaxies from which an IGC population might have been stripped (e.g., Forbes et al. 1997; Côté et al. 1998; Larsen et al. 2001; Gebhardt \& Kissler-Patig 1999; Coenda et al. 2009). In reality, however, such constraints are likely to be weak because of degenerate dependencies of globular cluster colors on galactocentric distances and host galaxy properties. A preponderance of blue IGCs could arise, for example, if the majority came from dwarf galaxies that were tidally disrupted $o r$ if they were stripped from the outer halos of giant galaxies whose globular clusters are bluer on average than their counterparts closer to the galaxy center (Geisler et al. 1996; Rhode \& Zepf 2004; Bassino et al. 2006; Tamura et al. 2006). Nevertheless, if the globular clusters are found to have a distinctive color distribution then this could be an important clue to their origin.

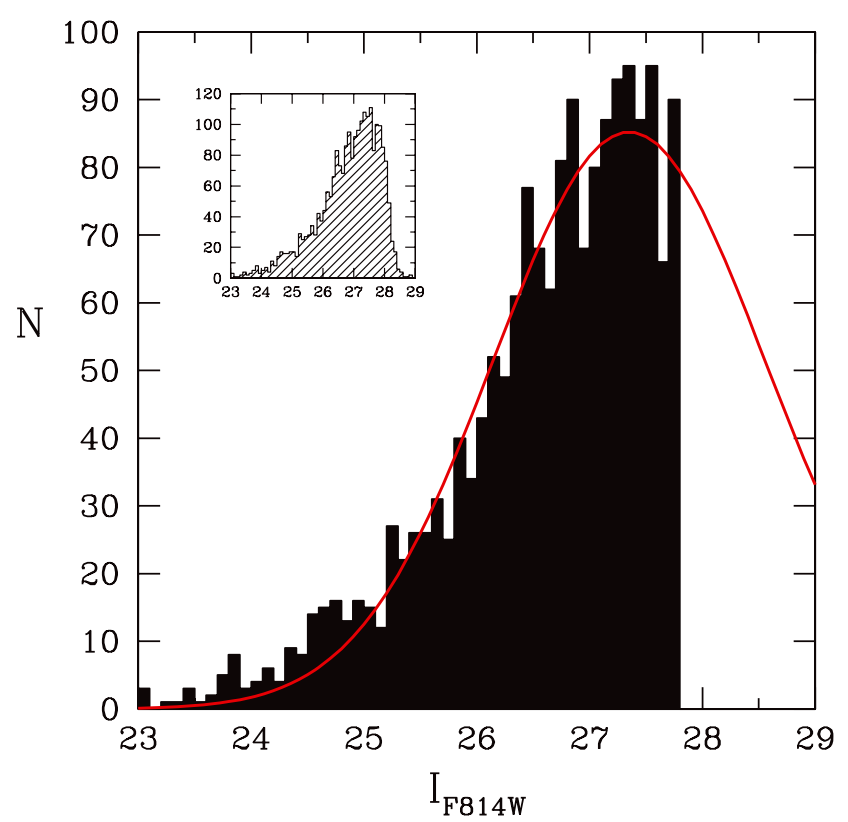

Fig. 13. The observed luminosity function of globular clusters in the core of A1185. The data have been corrected for incompleteness and the expected number of contaminating foreground/background objects have been removed based on the averaged counts at each magnitude from the four control fields. The luminosity function data are shown only to $I_{F 814 \mathrm{~W}} \simeq 27.8 \mathrm{mag}$ because beyond that incompletenss corrections become too large (more than a factor of two) to be reliable. The red curve is the best-fitting Gaussian luminosity function with a peak luminosity at $I_{F 814 W}=27.3 \mathrm{mag}$ and a standard deviation $\sigma=1.2$, as discussed in the text. The observed luminosity function is clearly consistent with the standard GCLF observed in many galaxies. The inset shows the raw luminosity function of globular cluster candidates without any corrections for background contamination or incompleteness.

Artificial star experiments show that matched detection of objects added to both the $F 555 \mathrm{~W}$ and $F 814 \mathrm{~W}$ images is $100 \%$ complete to $I_{F 814 W} \simeq 26.5 \mathrm{mag}$, as the shallower $F 555 \mathrm{~W}$ observations result in fewer detections at fainter magnitudes. To minimize the effect of this incompleteness on the inferred color distribution of globular clusters, we consider only those objects brighter than this magnitude limit.

Figure 14 shows the observed color distribution of globular clusters in the A1185 core field. These colors are quite similar to those of globular clusters associated with galaxies (Gebhardt \& Kissler-Patig 1999; Peng et al. 2006; Forbes et al. 2007), skewed strongly toward bluer (more metal-poor) colors. The peak in the color distribution occurs at $V-I \simeq 1.05$, in excellent agreement with the results of Gebhardt \& Kissler-Patig (1999), who found peak colors around $V-I \simeq 0.94-1.13$ depending on host galaxy luminosity. Although it is impossible to distinguish between IGCs and halo globular clusters in the HST images, it is clear that if IGCs are present then their colors cannot be grossly different from those of normal globular clusters. The colors are predominantly blue, as expected if IGCs are the surviving orphans of disrupted dwarf galaxies or were stripped from the outer halos of larger galaxies.

Figure 14 hints at possible bimodality or asymmetry of the globular cluster color distribution. To quantify this impression, we used the KMM mixture mode algorithm (McLachlan \& Basford 1988) as implemented by Ashman et al. (1994). KMM uses a maximum likelihood estimator to determine the 


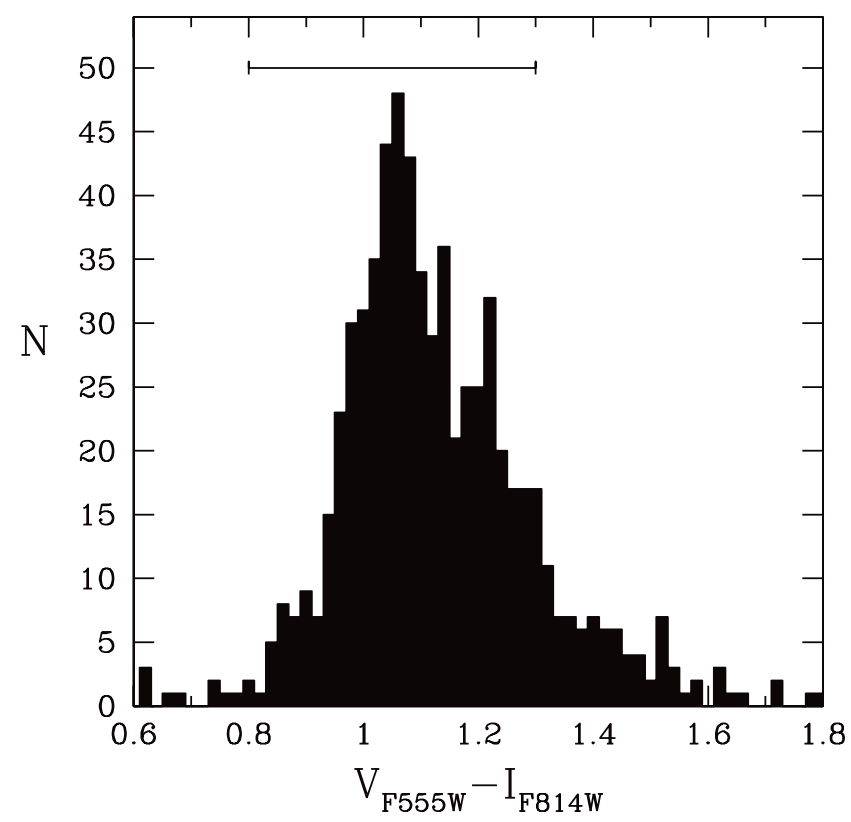

Fig. 14. The observed $V-I$ color distribution of globular clusters in the core of A1185. The bar indicates typical colors of globular clusters in the sample of Gebhardt \& Kissler-Patig (1999), corresponding to the $F W H M$ of their color distribution.

best fitting mixture of one or more Gaussian components to an observed distribution and estimates the mean and fraction of data points belonging to each component. Because outliers can adversely affect the KMM results (see Ashman et al. 1994, for a discussion) we restricted our analysis to the 616 clusters with colors in the range $0.8 \leq V-I \leq 1.4$ and $I_{F 814 W} \leq 26.5 \mathrm{mag}$. Assuming homoscedasticity (i.e., the same variance for each subpopulation), the KMM algorithm strongly rejects (at a confidence level of more than $99 \%$ ) the hypothesis that the color distribution seen in Fig. 14 is adequately described by a single Gaussian. The best fitting model has two Gaussian components, with roughly $2 / 3$ of globular clusters belonging to a blue subpopulation with a mean color $\langle V-I\rangle \simeq 1.04$ and $1 / 3$ belonging to a redder subpopulation with $\langle V-I\rangle \simeq 1.23$.

Given the detection of distinct blue and red subpopulations, we looked for evidence of the so-called "blue tilt" in the colormagnitude relation (Fig. 9), an observed tendency for blue globular clusters to become progressively redder at brighter magnitudes (e.g., Harris et al. 2006; Strader et al. 2006; Peng et al. 2009; Blakeslee et al. 2010; Mieske et al. 2010). In principle, the strength of the blue tilt, which is usually interpreted as a massmetallicity relation, could provide clues to the origin of the globular clusters in the center of A1185. As noted by Mieske et al. (2010), for example, the slope of the blue tilt in the Virgo and Fornax clusters of galaxies varies as a function of host galaxy luminosity and galactocentric distance.

For this purpose, we divided the sample of 616 globular clusters satisfying the aforementioned color and magnitude criteria into four luminosity bins containing equal numbers of objects. The KMM algorithm was then used to find the best fitting blue and red peaks in the color distribution for each luminosity bin (again assuming homoscedasticity) with $V-I=1.04$ and 1.23 chosen as the initial guess for their locations. Within each luminosity bin, the color distribution is well fitted by a mixture of two Gaussians. Because the KMM analysis does not provide a direct estimate of the uncertainties in the derived quantities, bootstrap

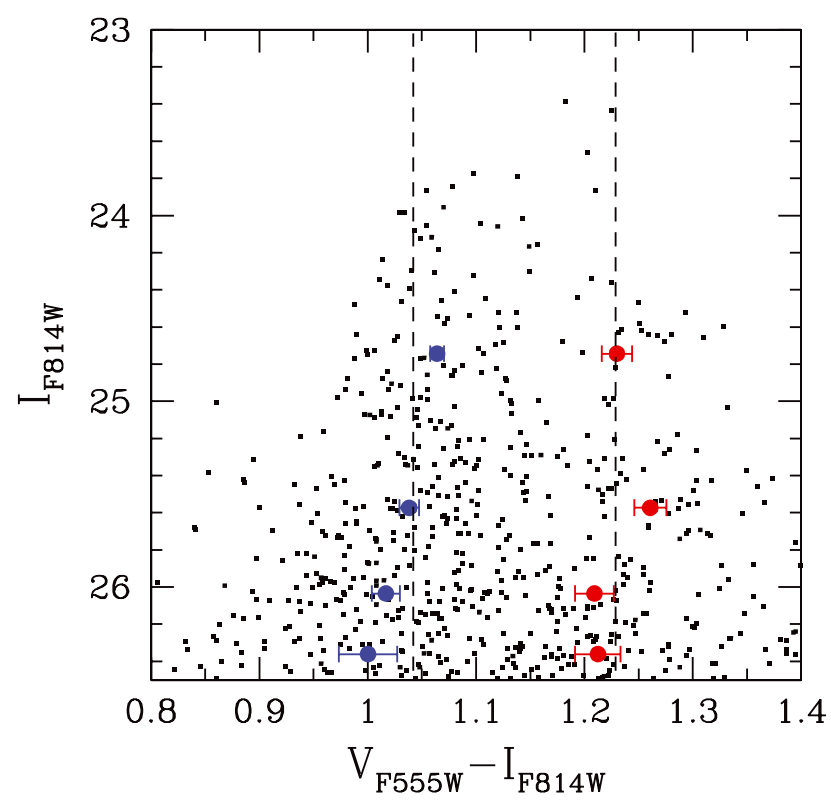

Fig. 15. Color-magnitude diagram for 616 globular clusters in the core of A1185 within the range $0.8 \leq V-I \leq 1.4$ and $I_{F 814 W} \leq 26.5$ mag. The blue and red points indicate the locations of the blue and red peaks determined by KMM fitting of the color distribution in four different luminosity bins to a model with two Gaussian components, as described in the text. Uncertainties in the peak locations were calculated via bootstrap resampling. The dashed lines denote the locations of the blue and red peaks found from KMM analysis for the entire sample of 616 clusters. A correlation between color and magnitude is apparent for the blue globular clusters (dubbed the "blue tilt") while no such trend is seen for the red clusters.

resampling was used to estimate the variance in the mean colors of the blue and red subpopulations. Results are shown in Fig. 15. Despite the relatively weak sensitivity of $V-I$ colors to metallicity (compared to the longer wavelength baselines provided by $g-z$ or $B-I$ used in most previous studies) a blue tilt is apparent. No obvious trend is seen for the red clusters. These visual impressions are confirmed by statistical analysis, which yields a Pearson correlation coefficient $r=-0.99$ (highly significant) for the color-magnitude relation of the blue subpopulation but only $r=-0.45$ (not significant) for the red subpopulation. The slope of the best fitting linear regression to the blue tilt seen in Fig. 15 is $\mathrm{d}(V-I) / \mathrm{d} I \simeq-0.039 \pm 0.013$, with the uncertainty estimated from bootstrap resampling. This slope is within the range found in previous studies. Given the sizable uncertainties, however, we refrain from further analysis or interpretation of these results.

\section{Discussion}

The results presented here show that the $\sim 1300$ globular clusters found in our ACS field are indistinguishable, at least in terms of their optical colors and luminosities, from the metalpoor globular cluster populations observed in hundreds of galaxies to date. Although a non-negligible fraction of these objects are undoubtedly chance projections of globular clusters residing in the extended halos of galaxies, we have argued based on detailed simulations that the majority are likely to be intergalactic. Nevertheless, given the uncertainties in these simulations we cannot confidently rule out the possibility that most or even all of the detected globular clusters might belong to galaxies rather than roaming the space between them. 
Confirmation that the candidate IGCs are truly intergalactic could be obtained in several ways. The most direct, but observationally challenging, method would be to measure velocities of these objects to determine whether they are indeed gravitationally bound to individual galaxies or whether they constitute an independent dynamical system in the core of A1185. In particular, the largest potential source of contaminating halo globulars, NGC 3550, has an observed radial velocity of $10520 \mathrm{~km} \mathrm{~s}^{-1}$ (Tonry 1985, gives velocities of 10447,10388 and $1100 \mathrm{~km} \mathrm{~s}^{-1}$ for each of the three components seen in Fig. 9), which differs by $\sim 1200 \mathrm{~km} \mathrm{~s}^{-1}$ from the $9300 \mathrm{~km} \mathrm{~s}^{-1}$ mean velocity of the A1185 system (Mahdavi et al. 1996), consistent with the spatial and kinematic substructure seen in this dynamically young cluster. Consequently, halo globular clusters associated with NGC 3550 could be readily distinguished from genuine IGCs moving in the gravitational potential of A1185 on the basis of their expected large velocity differences. The faint magnitudes of even the brightest IGC candidates in A1185 make such observations challenging, though not impossible, with 8-m to 10-m class telescopes.

Deep images of additional fields surrounding NGC 3550 could also help to determine whether IGCs are present. Globular clusters that are gravitationally bound to galaxies should generally be distributed symmetrically about their centers. If the number density of globular clusters in the central A1185 field shown in Fig. 1 is found to be significantly higher than that in other fields equidistant from NGC 3550, then this would support the idea that they are intergalactic. Several strategically placed fields might even allow the centroid of the putative IGC population to be localized.

Additional clues to the nature and origin of the globular cluster population in the heart of A1185 could come from measurements of their ages and metallicities. Although it is difficult at present to obtain such information spectroscopically because of the distance to A1185, a combination of optical and nearinfrared colors can provide an alternative measure of ages and metallicities (e.g., Puzia et al. 2002; Hempel et al. 2007; Carter et al. 2009).

Finally, we note that further study of A1185 may offer a tantalizing opportunity to glimpse the ongoing creation of IGCs. The merging galaxy system Arp 105 (see Fig. 1) is ejecting a long, narrow plume of material that probably includes extant globular clusters destined to become future intergalactic vagabonds, as well as newly created dwarf galaxies and possibly young star clusters (Duc \& Mirabel 1994). Similar tidal streams in other nearby galaxy clusters (e.g., Gregg \& West 1998; Calcáneo-Roldán et al. 2000; Rudick et al. 2009) would be excellent places to search for other IGCs. Deep, large-area surveys such as the ongoing Next Generation Virgo Cluster Survey (Ferrarese et al., in prep.) and the ACS Treasury Survey of the Coma Cluster (Carter et al. 2008) will also provide rich databases that can be searched for IGCs.

\section{Conclusions}

Using deep HST/ACS images, we have confirmed the presence of several thousand globular clusters in the heart of the rich galaxy cluster A1185, in a field that is not centered on any bright galaxy. Simulations suggest that the majority of these objects may be intergalactic, although the large uncertainties in these models make it impossible to reach firm conclusions about the reality or size of such a population. Our best estimate is that a total of $\sim 1600$ IGCs reside in the core ACS field, although we cannot rule out the possibility that they could be gravitationally bound to galaxies. The colors and luminosities of these objects are similar to those of metal-poor globular clusters found in galaxies, suggesting that - if they are indeed intergalactic - they probably formed in galaxies and were removed later by tidal stripping or other dynamical processes. We suggest that future observations, especially velocity measurements, could establish the true nature of the globular clusters in the center of A1185 as intergalactic wanders or galactic residents.

Acknowledgements. We thank Stefano Andreon and Jean-Charles Cuillandre for kindly providing their reduced CFH12k images of A1185, Sidney van den Bergh for enlightening discussions, and the anonymous referee for suggestions that helped to improve the paper. Support for programme HST-GO-9488 was provided by NASA through a grant from the Space Telescope Science Institute which is operated by the Association of Universities for Research in Astronomy, Inc., under NASA contract NAS 5-26555. This research made use of the NASA/IPAC Extragalactic Database (NED) which is operated by the Jet Propulsion Laboratory, California Institute of Technology, under contract with NASA. M.J.W. thanks the Herzberg Institute of Astrophysics for its hospitality during much of this work and acknowledges additional support from NSF grant AST 02-05960. A.J. acknowledges support from Fondecyt project 1095213, Anillo ACT86, BASAL CATA PFB-06, FONDAP CFA 15010003 and MIDEPLAN ICM Nucleus P07-021-F. Part of the work reported here was done at the Institute of Geophysics and Planetary Physics, under the auspices of the US Department of Energy by Lawrence Livermore National Laboratory in part under Contract W-7405-Eng-48 and in part under Contract DE-AC52-07NA27344.

\section{References}

Andreon, S., Cuillandre, J.-C., Puddu, E., \& Mellier, Y. 2006, MNRAS, 372, 60 Ashman, K. M., \& Zepf, S. E. 1998, Globular Cluster Systems (Cambridge: Cambridge University Press), 75

Ashman, K. M., Bird, C. M., \& Zepf, S. E. 1994, AJ, 108, 2348

Bassino, L. P., Faifer, F. R., Forte, J. C., et al. 2006, A\&A, 45, 789

Bekki, K., \& Yahagi, H. 2006, MNRAS, 372, 1019

Bertin, E., \& Arnouts, S. 1996, A\&AS, 117, 393

Blakeslee, J. P., Ph.D. Thesis, Massachusetts Insitute of Technology, 124

Blakeslee, J. P., Tonry, J. L., \& Metzger, M. R. 1997, AJ, 114, 482

Blakeslee, J. P., Anderson, K. R., Meurer, G. R., \& Bentez, N. 2003, in Astronomical Data Analysis Software and Systems XII, ed. H. E. Payne, R. I. Jedrzejewski, \& R. N. Hook (San Francisco: ASP), ASP Conf. Ser., 295, 257 Blakeslee, J. P., Cantiello, M., \& Peng. E. 2010, ApJ, 710, 51

Bassino, L. P., Cellone, S. A., Forte, J. C., \& Dirsch, B. 2003, A\&A, 399, 489

Brodie, J. P., \& Huchra, J. P. 1991, ApJ, 379, 157

Brodie, J. P., \& Strader, J. 2006, ARA\&A, 44, 193

Bromm, V., \& Clarke, C. 2002, ApJ, 566, L1

Calcáneo-Roldán, C., Moore, B., Bland-Hawthorn, J., Malin, D., \& Sadler, E. M. 2000, MNRAS, 314, 324

Carter, D., Goudfrooij, P., Mobasher, B., et al. 2008, ApJS, 176, 424

Carter, D., Smith, D. J. B., Percival, S. M., et al. 2009, MNRAS, 397, 695

Cen, R. 2001, ApJ, 560, 592

Coenda, V., Muriel, H., \& Donzelli, C. 2009, ApJ, 700, 1382

Côté, P., Marzke, R. O., \& West, M. J. 1998, ApJ, 501, 554

Côté, P., Blakeslee, J. P., Ferrarese, L., et al. 2004, ApJS, 153, 223

Duc, P.-A., \& Mirabel, I. F. 1994, A\&A, 289, 83

Elmegreen, B. G., 2010, ApJ, 712, L184

Fabian, A. C., Nulsen, P. E. J., \& Canizares, C. R. 1984, Nature, 310, 733

Fall, S. M., \& Rees, M. J. 1985, ApJ, 298, 18

Fall, M. S., \& Zhang, Q. 2001, ApJ, 561, 751

Fasano, G., \& Franceschini, A. 1987, MNRAS, 225, 155

Forbes, D. A., \& Forte, J. C. 2001, MNRAS, 322, 257

Forbes, D. A., Brodie, J. P., \& Grillmair, C. J. 1997, AJ, 113, 1648

Forbes, D. A. Proctor, R., Strader, J., \& Brodie, J. 2007, ApJ, 659, 188

Forte, J. C., Martinez, R. E., \& Muzzio, J. C. 1982, AJ, 87, 1465

Fruchter, A. S., \& Hook, R. N. 2002, PASP, 114, 144

Gebhardt, K., \& Kissler-Patig, M. 1999, AJ, 118, 1526

Geisler, D., Lee, M. G., \& Kim, E. 1996, AJ, 111, 1529

Graham, A. W., \& Driver, S. P. 2005. PASA, 22, 118

Gregg, M. D., \& West, M. J. 1998, Nature, 396, 549

Gregg, M. D., Drinkwater, M. J., Evstigneeva, E. et al. 2009, AJ, 137, 498

Griffen, B. F., Drinkwater, M. J., Thomas, P. A., Helly, J. C., \& Pimbblet, K. A. 2010, MNRAS, 405, 375

Harris, W. E. 1991, ARA\&A, 29, 543 
Harris, W. E. 2001, in Star Clusters: SAAS-FEE Advanced Course 28, ed. L. Labhardt, \& B. Binggeli (Berlin: Springer-Verlag), 223

Harris, W. E., \& van den Bergh, S. 1981, AJ, 86, 1627

Harris, W. E., Kavelaars, J. J., Hanes, D. A., Hesser, J. E., \& Pritchet, C. J. 2000, ApJ, 533, 137

Harris, W. E., Whitmore, B. C., Karakla, D., et al. 2006, ApJ, 636, 90

Hempel, M., Zepf, S., Kundu, A., Geisler, D., \& Maccarone, T. J. 2007, ApJ, 661,768

Hilker, M. 2003, in New Horizons in Globular Cluster Astronomy, ed. G. Piotto, G. Meylan, G. Djorgovski, \& M. Riello, ASP Conf. Ser., 296, 583 Janowiecki, S., Mihos, J. C., Harding, P., et al. 2010, ApJ, 715, 972

Jordán, A., West, M. J., Côté, P., \& Marzke, R. O. 2003, AJ, 125, 1642

Jordán, A., McLaughlin, D. E., Côté, P., et al. 2006, ApJ, 651, L25

Jordán, A., McLaughlin, D. E., Côté, P., et al. 2007, ApJS, 171, 101

Kravtsov, A. V., \& Gnedin, O. Y. 2005, ApJ, 623, 650

Lee, M. G., Park, H. S., \& Hwang, H. S. 2010, Science, 328, 334

Mackey, A. D., Huxor, A. P., Ferguson, A. M. N., et al. 2010, ApJ, 717, L11

Mahdavi, A., Geller, M. J., Fabricant, D. G., et al. 1996, AJ, 111, 64

McLachlan, G. J., \& Basford, K. E. 1988, Mixture Models: Inference and Application to Clustering (New York: M. Dekker)

McLaughlin, D. E., \& Fall, S. M. 2008, ApJ, 679, 1272

Merritt, D. 1984, ApJ, 276, 26

Mieske, S., Jordán, A., Côté, P., et al. 2010, ApJ, 276, 26

Mihos, J. C., Harding, P., Feldmeier, J., \& Morrison, H. 2005, ApJ, 631, L41

Muzzio, J. C., Martinez, R. E. \& Rabolli, M. 1984, ApJ, 285, 7

Peacock, J. A. 1983, MNRAS, 202, 615

Peebles, P. J. E. 1984, ApJ, 710, 1672

Peebles, P. J. E., \& Dicke, R. H. 1968, ApJ, 154, 891

Peng, E. W., Jordán, A., Côté, P., et al. 2006, ApJ, 639, 95
Peng, E. W., Jordán, A., Côté, P., et al. 2008, ApJ, 681, 197 Peng, E. W., Jordán, A., Côté, P., et al. 2009, ApJ, 703, 42 Peng, E. W., Ferguson, H. C., Goudfrooij, P., et al. 2011, ApJ, 730, 23

Press, W. H., Teukolsky, S. A., Vetterling, W. T., \& Flannery, B. P. 1997, Numerical Recipes in Fortran 77 (Cambridge: Cambridge University Press)

Puzia, T. H., Zepf, S. E., Kissler-Patig, M., et al. 2002, A\&A, 391, 453

Rhode, K. L., \& Zepf, S. E. 2001, AJ, 121, 210

Rhode, K. L., \& Zepf, S. E. 2004, AJ, 127, 302

Rudick, C. S., Mihos, J. C., Frey, L. H., \& McBride, C. K. 2009, ApJ, 699, 1518

Schlegel, D. J., Finkbeiner, D. P., Davis, M. 1998, ApJ, 500, 525

Schuberth, Y., Richtler, T., Bassino, L., \& Hilker, M. 2008, A\&A, 477, L9

Sérsic, J.-L. 1968, Atlas de Galaxias Australes (Cordoba: Observatorio Astronomico)

Sirianni, M. Jee, M. J., Benítez, N., et al. 2005, PASP, 117, 1049

Stetson, P. B. 1987, PASP, 99, 191

Strader, J., Brodie, J. P., Spitler, L., \& Beasley, M. A. 2006, AJ, 132, 2333

Tamura, N., Sharples, R. M. Arimoto, N., et al. 2006, MNRAS, 373, 601

Tonry, J. L. 1985, AJ, 90, 2431

van den Bergh, S. 1958, Obs., 78, 85

van den Bergh, S. 1975, ARA\&A, 13, 217

Vesperini, E., Zepf, S. E., Kundu, A., \& Ashman, A. 2003, ApJ, 593, 760

Villegas, D., Jordán, A., Peng, E. W., et al. 2010, ApJ, 717, 603

West, M. J. 1993, MNRAS, 265, 755

West, M. J., Côté, P., Jones, C., Forman, W., \& Marzke, R. O. 1995, ApJ, 453, L77

West, M. J., Côté, P., Marzke, R. O., \& Jordán, A. 2004 , Nature, 427, 31

White, R. E., 1987, MNRAS, 227, 185

Williams, B. F., Ciardullo, R., Durrell, P. R., et al. 2007, ApJ, 654, 835

Yahagi, H., \& Bekki, K. 2005, MNRAS, 344, 1334 